\title{
Quiste tímico inusual de mediastino posterior en un paciente con neurofibromatosis
}

\author{
Unusual thymic cyst of posterior mediastinum \\ in a patient with neurofibromatosis
}

\author{
Eric Narciso-Dircio, ${ }^{*}$ Liliana Denisse Valencia-Sánchez, ${ }^{*}$ Juan Carlos Vázquez-Minero*
}

*Instituto Nacional de Enfermedades Respiratorias Ismael Cosío Villegas, Ciudad de México.

\begin{abstract}
RESUMEN. Los tumores del timo son los más frecuentes en el mediastino anterior. Los quistes mediastinales congénitos se presentan de 3 a $6 \%$. Su localización posterior es extremadamente rara y por ende, la clínica que los acompaña; sin embargo, debe considerarse entre los diagnósticos diferenciales, tal como sucede con el caso que se presenta a continuación en una paciente con antecedente de neurofibromatosis en un instituto de referencia. Se trata de una mujer de 53 años de edad con un quiste de mediastino posterior sometida a resección por toracotomía, que en el estudio definitivo por patología se trató de un quiste tímico, con buenos resultados y con seguimiento sin recidiva.
\end{abstract}

Palabras clave: Quiste tímico, quiste mediastino, mediastino posterior, quiste neurofibromatosis.

\section{INTRODUCCIÓN}

Los tumores del timo son los más frecuentes a nivel del mediastino anterior, siendo el timoma el más frecuente. ${ }^{1}$ Los quistes mediastinales congénitos representan de 3 a $6 \%$ de todos los tumores mediastinales y de 10 a $18 \%$ de todos los que son reportados radiológicamente. ${ }^{2}$

Los quistes del timo se presentan en menos de $1 \%$ de los tumores quísticos mediastinales y pueden ubicarse desde el ángulo de la mandíbula hasta el diafragma. Se clasifican de acuerdo con su origen en congénitos o adquiridos. Los quistes congénitos son uniloculares y se caracterizan por

\section{Correspondencia:}

\section{Dr. Eric Narciso-Dircio}

Instituto Nacional de Enfermedades Respiratorias

Ismael Cosío Villegas, Ciudad de México.

Correo electrónico: nade8686@hotmail.com

Trabajo recibido: 12 -XII-2019; aceptado: 08-V-2020.

Citar como: Narciso-Dircio E, Valencia-Sánchez LD, Vázquez-Minero JC. Quiste tímico inusual de mediastino posterior en un paciente con neurofibromatosis. Neumol Cir Torax. 2020;79(3):171-175. https://dx.doi. org/10.35366/96652

\begin{abstract}
Thymus tumors are the most frequent in the anterior mediastinum. Congenital mediastinal cysts occur 3 to $6 \%$. Their posterior location is extremely rare, and therefore the clinic that accompanies them; however, it should be considered among the differential diagnoses, as is the case in the case presented below in a patient with a history of neurofibromatosis in a reference institute. This is a 53-year-old woman with a posterior mediastinal cyst, undergoing thoracotomy resection, which in the definitive pathology study was a thymic cyst, with good results and with follow-up without recurrence.
\end{abstract}

Keywords: Thymic cyst, mediastinal cyst, posterior mediastinum, neurofibromatosis cyst.

una pared delgada y translúcida. Los quistes adquiridos son quistes tímicos multiloculares con paredes gruesas casi siempre asociadas con proceso inflamatorio y pueden surgir en asociación con neoplasias como timomas, linfomas o tumores de células germinales. Los quistes tímicos también se pueden ver en el mediastino anterior después de la radioterapia de la enfermedad de Hodgkin. ${ }^{3}$

El timo se origina a partir de la tercera y cuarta hendiduras de los arcos branquiales, posee células de las tres capas embrionarias y migra desde el ángulo mandibular hacia el mediastino anterior y superior. ${ }^{4}$ Como consecuencia de los defectos de la migración durante la embriogénesis se puede encontrar tejido tímico ectópico o aberrante en todo este trayecto, ya sea en continuidad o separado del timo principal. Por lo tanto, la localización definitiva del tejido tímico puede ser diferente, se puede extender hacia la región posterior a la vena cava superior al lado derecho o hacia la izquierda en forma paralela al arco aórtico. Incluso puede extenderse al mediastino medio e incluso al mediastino posterior.

Los quistes congénitos pueden estar presentes en mediastino posterior en particular en aquellos casos que tienen un desplazamiento vertebral asociado y síntomas neurológicos. ${ }^{2}$ 
Los tumores del timo no tienen signos clínicos patognomónicos y hasta $30 \%$ de los pacientes pueden ser asintomáticos. La lesión por estos tumores se hace por medio de la tomografía computarizada (TC) de tórax en 50\% de los casos de forma incidental. La biopsia no está indicada de rutina, a menos que existan dudas diagnósticas y se esté sospechando un tumor de origen distinto (metástasis pulmonares, tumor de células germinales, linfomas y/o bocio intratorácico, entre otros. ${ }^{5,6}$

Por su naturaleza benigna, la mayoría de los quistes asintomáticos requieren seguimiento imagenológico. Los quistes sintomáticos sí requieren tratamiento. El drenaje del quiste con aguja guiada por imagen puede realizarse cuando los síntomas son leves o si se requiere una evaluación citológica. Los quistes con síntomas más severos o quistes de localización no habitual o multiloculados requieren resección quirúrgica, la cual se puede realizar con una toracotomía o con un procedimiento videotoracoscópico, pero a veces se puede requerir una esternotomía. El procedimiento a elegir habitualmente se lleva a cabo en la individualización de cada caso en particular.

\section{PRESENTACION DEL CASO}

Se trata de mujer de 53 años de edad, con diagnóstico de neurofibromatosis (enfermedad de von Recklinghausen) desde los seis años de edad, en seguimiento, y antecedentes quirúrgicos al respecto con resección de neurofibroma a nivel de columna lumbar a los seis años, posteriormente otro en muslo izquierdo a los 12 años de edad.

Tres meses de evolución con dolor torácico izquierdo de inicio posterior irradiándose a hombro izquierdo, de intensidad moderada de forma progresiva, se acompaña de disnea

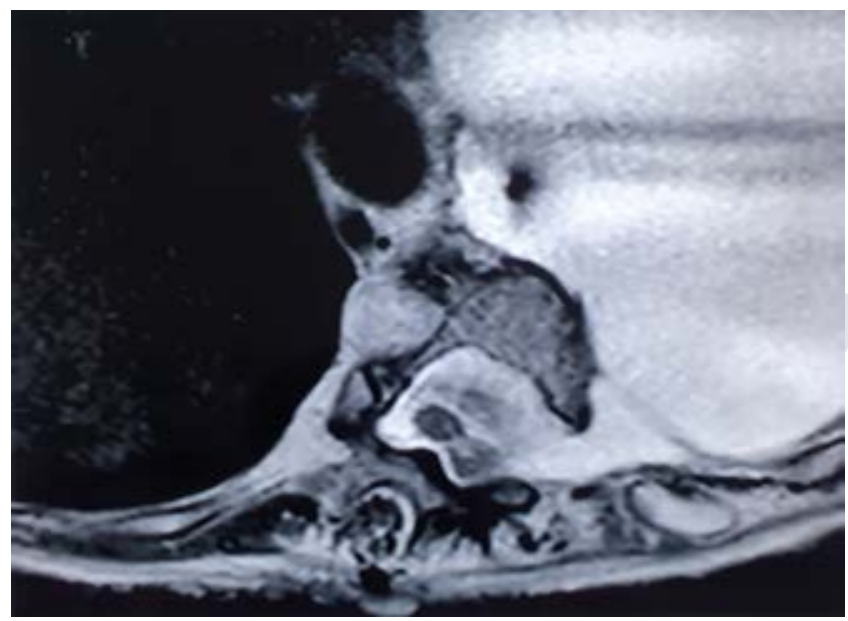

Figura 1: Resonancia magnética, secuencia T1 axial a nivel de T8 que muestra aparente comunicación medular con tumor en hemitórax izquierdo inicialmente catalogado como quiste meníngeo.

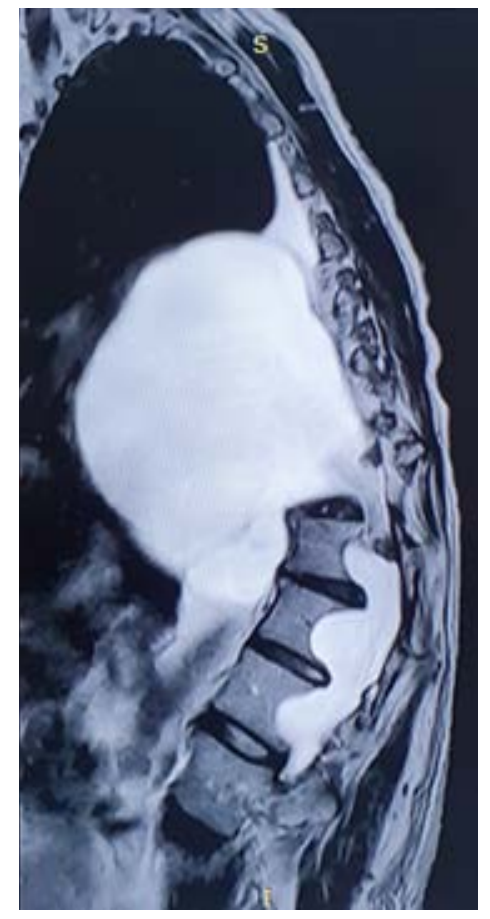

Figura 2:

Resonancia magnética, secuencia T1 sagital con tumor quístico de mediastino posterior con compresión a pulmón izquierdo y diafragma en íntima relación con columna.

a medianos esfuerzos, tos con expectoración blanquecina, escalofríos de predominio nocturno, astenia y adinamia. Acudió con neurólogo por exacerbación de sintomatología y solicitó radiografía simple de tórax y resonancia magnética torácica de columna (Figuras 1 y 2) integrando probable quiste meníngeo, sin manifestaciones clínicas neurológicas relacionadas con déficit motor ni sensitivo, por lo que es referida a nuestro instituto. A su ingreso presentó dolor torácico pleurítico posterior izquierdo, se irradia a cara anterior en esta ocasión, disnea de esfuerzos menores, cuadro semejante al inicial, se solicitó tomografía de tórax que reportó tumor del mediastino posterior de contenido líquido en comunicación probable a médula espinal, dismorfia a nivel de séptima y octava vértebra torácica, correspondiendo a impresión diagnóstica inicial de referencia.

Se sometió a tratamiento quirúrgico realizándose abordaje mediante toracotomía posterolateral identificando tumor quístico de aproximadamente $12 \mathrm{~cm}$ en su eje transverso y $10 \mathrm{~cm}$ en el longitudinal con pared de $5 \mathrm{~mm}$ que contenía líquido transparente sin septos, en comunicación a espacio intervertebral entre T7 y T8, con deformidad de estos cuerpos a expensas de defecto intervertebral de $2 \mathrm{~cm}$ de diámetro sin visualizar raíces nerviosas, se drenó contenido por aspiración y resecó pared de tumor. Se realizó colocación de colgajo de pleura parietal a nivel de defecto, así como gelatina en esponja de uso quirúrgico y cianoacrilato como manejo quirúrgico de fístula de líquido cefalorraquídeo (LCR). Pulmón con expansión óptima (Figura 3). 
La paciente presentó evolución satisfactoria con reinicio de dieta el mismo día de la intervención, con tratamiento médico preventivo ante una probable fístula de LCR, presentando disminución progresiva de drenaje por sonda endopleural, rehabilitación respiratoria integral, con retiro de drenaje torácico a los siete días del procedimiento, egresando a su domicilio sin sintomatología neurológica secundaria.

Se obtiene reporte de anatomía patológica que concluye como tumor compatible con quiste tímico, presentando pared con tejido fibroso e infiltrado linfoide con corpúsculos de Hassall y marcadores de inmunohistoquímica positivos: calretinina $(+++/+++)$ y citoqueratina $5 / 6(+++/+++)$ en células mesoteliales reactivas (revestimiento externo de la pared). Controles negativos: PS-100, enolasa neuronal específica, CK AE1/AE3 y EMA (Figuras 4 y 5).

Se realizó seguimiento posoperatorio sin complicaciones con adecuada evolución a los tres meses.

\section{DISCUSIÓN}

Como primer punto se mencionan las particularidades en relación con los quistes de naturaleza meníngea, ya que el contexto del preoperatorio en el abordaje clínico y de imagenología nos dio la pauta para integrar una conducta quirúrgica acorde a este diagnóstico, y que se descartó por lo que a continuación se describe. Los quistes aracnoideos corresponden a $1 \%$ de los tumores espinales y se definen como un divertículo de la duramadre, la aracnoides o de la vaina de una raíz nerviosa que resultan en la acumulación de líquido similar al LCR en el espacio extradural o intradural. Se encuentran dorsales a la médula espinal, tal como el caso que presentamos, y existen reportes en posición posterolateral y anterior. El conducto raquídeo a nivel torácico es relativamen-

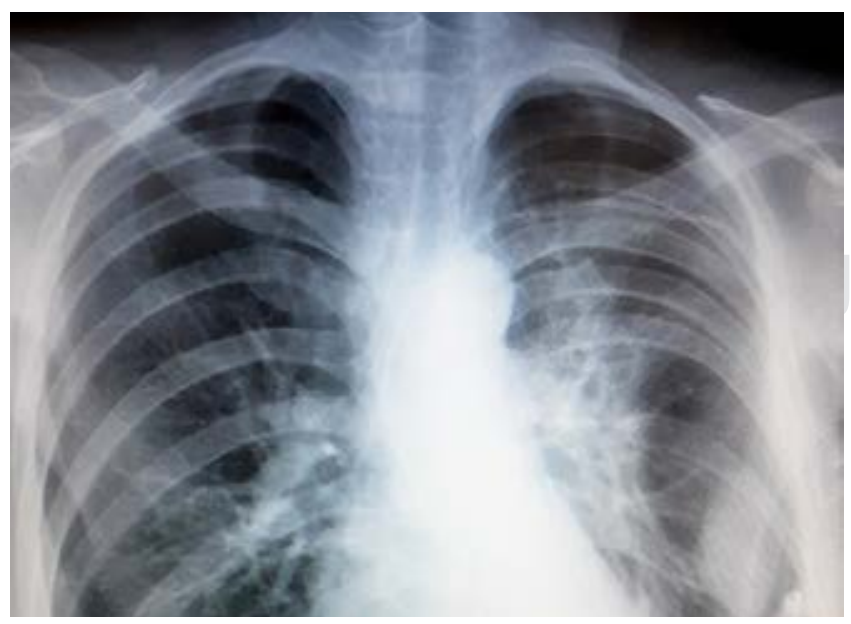

Figura 3: Radiografía posteroanterior de tórax posoperatoria con expansión pulmonar adecuada y mediastino en posición central.

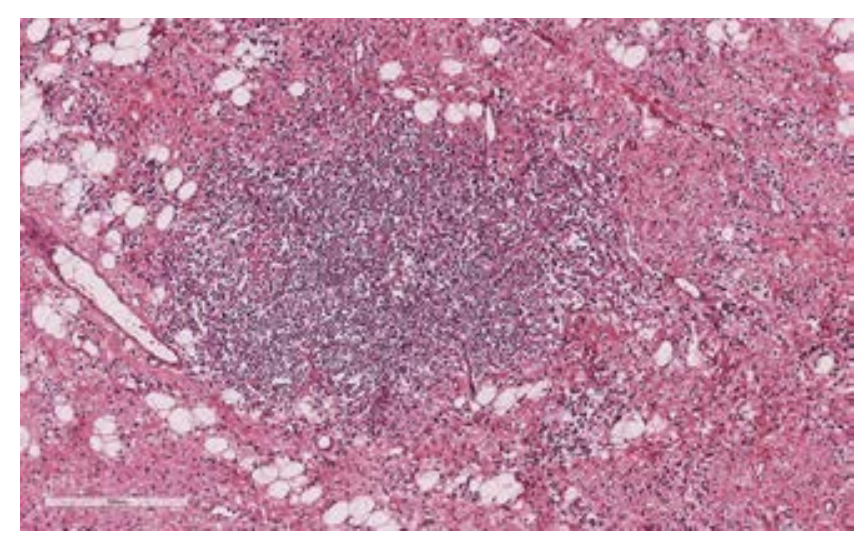

Figura 4: Corte histopatológico con aumento de 10x, tinción hematoxilina y eosina, que muestra en el centro un cúmulo de linfocitos típicos en relación a médula de timo, así como tejido conjuntivo y vasos sanguíneos abundantes.

te pequeño en su diámetro, por lo que los quistes presentan sintomatología con mayor frecuencia. Son tumores benignos que se presentan con frecuencia en la región torácica media y baja (65\%), en la columna lumbar y lumbosacra (13\%), toracolumbar (12\%), sacra (7\%) y cervical (3\%). Los síntomas que presentan son fluctuantes y son consecuencia de cambios en la presión hidrostática del LCR que a su vez originan cambios de presión dentro del quiste, por lo que se exacerban con cambios posturales, ejercicio físico y/o con maniobras de Valsalva. En su histología existe tejido fibrocartilaginoso, células meningoepiteliales, células inflamatorias y en gran parte de los casos tejido aracnoideo. En cuanto a la inmunohistoquímica, la enolasa neuronal específica, la proteína S 100 y el antígeno de membrana epitelial (EMA) son contundentes asociados al análisis estructural. Características que no se detectaron en la revisión de los cortes de la pieza quirúrgica en este caso, y que nos llevó a profundizar el contexto de los quistes tímicos tal como se integró finalmente.

Los quistes tímicos posteriores son prácticamente escasos en la literatura. Incluso se han descrito con relativa mayor frecuencia a nivel cervical en relación con los primeros, tal como lo describe Bothale en su estudio. ${ }^{8}$ En nuestro instituto es el primer caso en 15 años. Takeda ${ }^{9}$ publicó en su serie que la prevalencia de quistes mediastinales es mayor en adultos que en niños. $14.7 \%$ versus $7 \%$. De 105 pacientes, 30 fueron tímicos y $40 \%$ de ellos sintomáticos, tratados todos de forma quirúrgica.

La presentación clínica de los quistes tímicos es inespecífica y está en relación al efecto compresivo por el tamaño del tumor hacia las estructuras adyacentes. En el caso que describimos hubo compresión al pulmón y el diagnóstico se realizó por medio de estudio histopatológico tras revisiones repetidas debido a la topografía inicial del tumor en el análisis de estudios de gabinete, los hallazgos transoperatorios y el diagnóstico inicial desde la infancia. 
En un estudio publicado por Esme ${ }^{10}$ en Turquía se analizaron los resultados clínicos y quirúrgicos sobre tumores quísticos mediastinales y en dos de siete pacientes con quistes tímicos eran asintomáticos; dos mostraron disnea, dos tenían tos y tres presentaban angina; cinco fueron sometidos a TC de tórax para la evaluación de masas mediastínicas anterior-superiores detectadas en la radiografía de tórax inicial. Los quistes tímicos se ubicaron en el mediastino anterior en cuatro pacientes y en el mediastino medio sólo en tres.

Zhonggao ${ }^{1}$ en China realizó un análisis comparativo sobre los hallazgos tomográficos característicos en timoma y en quistes de mediastino, demostrando el predominio de los quistes en hombres en una relación 1:3. Con un promedio de 38.1 unidades Hounsfield (UH). El caso presentado muestra densidades entre 2 y $20 \mathrm{UH}$.

El estudio de Vargas ${ }^{11}$ refiere que mediante estudio tomográfico es casi imposible distinguir las características que diferencian a los distintos tumores quísticos; sin embargo, da la pauta para que mediante la resonancia magnética (RMN) se precise la anatomía de forma más íntima con las estructuras adyacentes y se oriente al diagnóstico. En nuestro caso las características por RMN, la localización y la relación anatómica estrecha con la columna vertebral nos hicieron pensar en un quiste meníngeo torácico; sin embargo, no hubo alteraciones clínicas neurológicas en el posquirúrgico relacionadas con descompresión subdural.

El abordaje que se utilizó de manera electiva fue la toracotomía posterolateral, ya que permite una adecuada exposición de estructuras ante un tumor de volumen considerable

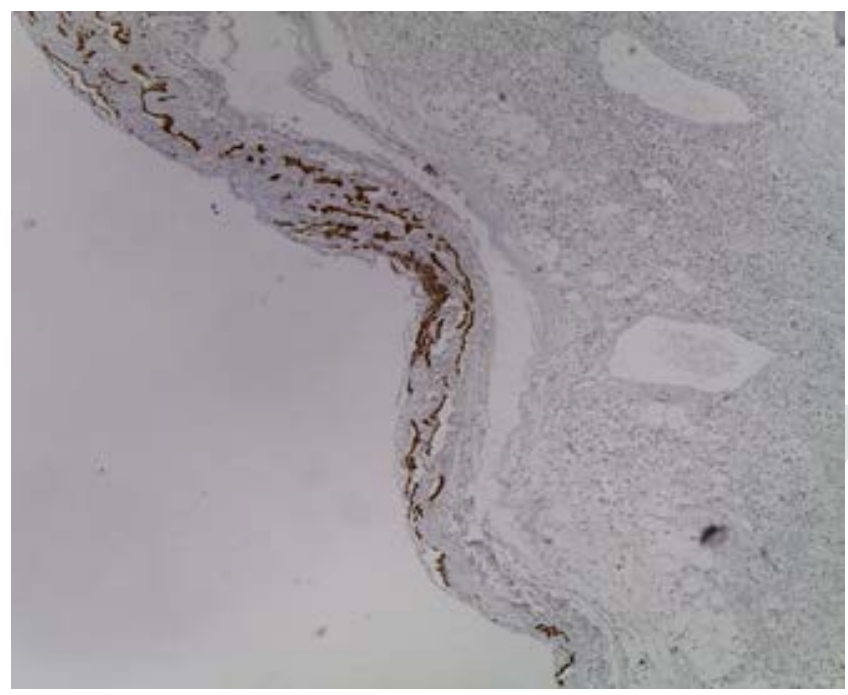

Figura 5: Corte aumento 10x que muestra inmunohistoquímica a citoqueratina 5/6 reactiva en células de revestimiento mesotelial así como proteína $\mathrm{S} 100$ y enolasa neuronal específica negativas. Ausencia de tejido perineural. e íntimamente relacionado a estructuras intervertebrales y óseas en mediastino posterior, permitiendo de esta forma evaluar con visión directa las características morfológicas del sitio en cuestión. De esta forma, ante el diagnóstico probable que se contemplaba, nos permitió cubrir el defecto con parche de pleura parietal y aislar una probable comunicación medular hacia resto de la cavidad torácica, lo que condujo a que la producción de líquido pleural durante la recuperación se comportara de manera habitual sin incidentes ni complicaciones, lo cual nos permitió retirar el drenaje de forma oportuna con adecuada recuperación.

En una revisión sobre quistes mediastinales se demostró que el tratamiento quirúrgico conlleva a una morbilidad aceptable, pero con resultados excelentes en casos sintomáticos. ${ }^{9}$ Gürsoy ${ }^{12}$ indicó tratamiento resectivo tomando en cuenta el tamaño tumoral, la localización y la penetración en estructuras circundantes e incluso para diagnóstico preciso. 26 casos fueron sometidos a toracotomía, uno a mediastinoscopia por quiste unilocular anterior, y uno a resección videotoracoscópica, con resultados adecuados a un año de seguimiento. Nuestro caso lleva seguimiento de seis meses sin recidiva.

Las características histológicas que cumple un quiste del timo son particulares, y deben tenerse en consideración los corpúsculos de Hassall, que son células epiteliales reticulares eosinofílicas dispuestas en la médula del timo, aunque no siempre se presentan en todos los casos. Asimismo, pueden observarse cúmulos de linfocitos dispuestos en un área, traduciendo su disposición de maduración. Su inmunohistoquímica debe tener presentes CD3 y citoqueratina para evaluar la densidad del epitelio mesotelial de revestimiento, EMA, PS 100 es negativo.

El caso en particular no cumple con las características firmes descritas en la literatura para apoyar que se tratase de un quiste meníngeo, excepto por las de imagenología. El comportamiento clínico neurológico fue prácticamente nulo en una topografía medular donde los síntomas florecen en relación con la sensibilidad y motricidad de extremidades ante compresión radicular y/o medular. Asimismo, el componente respiratorio por restricción fue endeble a pesar del tamaño del tumor.

Por otro lado, es importante señalar que la sensibilidad y especificidad de los marcadores inmunohistoquímicos son de gran impacto para establecer la naturaleza del tejido resecado. En este caso, además de tener disponibles los marcadores, se presentaron los hallazgos histológicos complementarios que tienen sustancial relación con tejido que proviene del timo; asimismo, exclusión de marcadores de naturaleza nerviosa como la enolasa neuronal específica EMA, PS 100, y la ausencia de revestimiento meningoepitelial característico y propio descartó la impresión diagnóstica inicial respecto a un probable quiste meníngeo concretando así el diagnóstico final. 


\section{CONCLUSIONES}

Los quistes de timo son poco frecuentes en la población general; sin embargo, no debe ocultarse su consideración para establecer diagnósticos diferenciales en tumores de mediastino, independientemente de su localización e inclusive en quistes de mediastino posterior, tal como el caso que aquí presentamos. Las conductas diagnóstica y terapéutica deben ser muy particulares a fin de individualizar cada una de ellas. Es necesaria la comunicación interdisciplinaria en la atención del paciente, siendo contundente el análisis histopatológico.

\section{Agradecimientos}

Agradecimientos al Servicio de Anatomía Patológica del Instituto Nacional de Enfermedades Respiratorias Ismael Cosío Villegas por su amable colaboración con el caso.

\section{REFERENCIAS}

1. Zhonggao J, YiJiao W, Yongfeng W, Zhitao P, Jun W, Diansheng L, et al. Multislice computed tomography performance in differential diagnosis of high-density thymic cyst and thymoma in lesions less than $3 \mathrm{~cm}$. Thorac Cancer. 2018;9(10):1300-1304. Available in: https:// doi.org/10.1111/1759-7714.12840

2. Jennings S, Stuklis RG, Chan J, Kearney D. Successful giant thymic cyst removal: case report and review of the literature. Heart Lung Circ. 2015;24(7):e89-e92. Available in: https://doi.org/10.1016/j. hlc.2015.02.013

3. Juanpere S, Cañete N, Ortuño P, Martínez S, Sanchez G, Bernado L. A diagnostic approach to the mediastinal masses. Insights Imaging.
2013;4(1):29-52. Available in: https://doi.org/10.1007/s13244-0120201-0

4. Alarcón ER, García BC. Caso clínico-radiológico. Rev Chil Pediatr. 2013;84(6):681-684.

5. Nguyen A, Habib H, Awad WI. Giant thymic cyst mimicking a unilateral pleural effusion. Thorax. 2017;72(11):1060-1061. Available in: https:// doi.org/10.1136/thoraxjnl-2016-209460

6. Buitrago-Ramírez MR, Mantilla-Gaviria HJ, Carreño-Dueñas JA. Tumores del timo: Experiencia del Instituto Nacional de Cancerología de Colombia. Rev Colomb Cancerol. 2019;23(3):92-98. Disponible en: https://doi.org/10.35509/01239015.17

7. Bouma W, Klinkenberg TJ, Van-De-Wauwer C, Timens W, Mariani MA. Removal of a giant intrathoracic cyst from the anterior mediastinum. J Cardiothorac Surg. 2014;9:152. Available in: https://doi.org/10.1186/ s13019-014-0152-2

8. Bothale KA, Mahore SD, Karmarkar P, Dongre T. IMAGES: ectopic thymic cyst: a rare case report. Indian J Pathol Microbiol. 2015;58(1):118119. Available in: https://doi.org/10.4103/0377-4929.151207

9. Takeda SI, Miyoshi S, Minami M, Ohta M, Masaoka A, Matsuda H. Clinical spectrum of mediastinal cysts. Chest. 2003;124(1):125-132. Available in: https://doi.org/10.1378/chest.124.1.125

10. Esme H, Eren S, Sezer M, Solak O. Primary mediastinal cysts: clinical evaluation and surgical results of 32 cases. Tex Heart Inst $\mathrm{J}$. 2011;38(4):371-374.

11. Vargas D, Suby-Long T, Restrepo CS. Cystic lesions of the mediastinum. Semin Ultrasound CT MR. 2016;37(3):212-222. Disponible en: https://doi.org/10.1053/j.sult.2015.12.005

12. Gürsoy S, Ozturk A, Ucvet A, Erbaycu AE. Lesiones quísticas primarias y benignas del mediastino en el adulto: espectro clínico y tratamiento quirúrgico. Arch Bronconeumol. 2009;45(8):371-375. Disponible en: https://doi.org/10.1016/j.arbres.2009.01.006

Conflicto de intereses: Los autores declaran no tener conflicto de intereses. 